\title{
Biochemical Persistence of Prostate-specific Antigen after Robot-assisted Laparoscopic Radical Prostatectomy: Tumor localizations using PSMA PET/CT imaging
}

\author{
Dennie Meijer ${ }^{1,2}$, Maarten L. Donswijk ${ }^{3}$, Yves J.L. Bodar ${ }^{1,2}$, \\ Pim J. van Leeuwen ${ }^{4}$, Henk G. van der Poel ${ }^{4}$, Wouter V. Vogel ${ }^{3}$, \\ Jakko A. Nieuwenhuijzen ${ }^{1,4}$, N. Harry Hendrikse ${ }^{2,5}$, \\ Daniela E. Oprea-Lager ${ }^{2}$, André N. Vis ${ }^{1,4}$
}

\footnotetext{
${ }^{1}$ Amsterdam University Medical Center, VU University, Department of Urology, Prostate Cancer Network The Netherlands, Amsterdam, The Netherlands

${ }^{2}$ Amsterdam University Medical Center, VU University, Department of Radiology \& Nuclear Medicine, Cancer Center Amsterdam, Amsterdam, The Netherlands

${ }^{3}$ The Netherlands Cancer Institute, Department of Nuclear Medicine, Amsterdam, The Netherlands

${ }^{4}$ The Netherlands Cancer Institute, Department of Urology, Prostate Cancer Network The Netherlands, Amsterdam, The Netherlands

${ }^{5}$ Amsterdam University Medical Center, VU University, Department of Clinical Pharmacology and Pharmacy, Amsterdam, The Netherlands
}

\author{
Corresponding author \\ Dennie Meijer, MD \\ ORCID 0000-0002-8298-575X \\ De Boelelaan 1117, 1081 HV Amsterdam \\ Telephone: +31-20-4443289 / FAX: +31-20-4446031 \\ d.meijer2@amsterdamumc.nl
}

Short running title: PSMA PET/CT imaging in patients with $B C P$ 


\section{ABSTRACT}

Since the introduction of radiolabeled prostate-specific membrane antigen (PSMA) positron emission tomography/computed tomography (PET/CT), the ability to visualize recurrent prostate cancer has improved substantially. However, the diagnostic accuracy of radiolabeled PSMA $\mathrm{PET} / \mathrm{CT}$ in patients with biochemical persistence (BCP; i.e., persistently measurable prostatespecific antigen (PSA)-values after robot-assisted laparoscopic radical prostatectomy (RARP)) is largely lacking. Therefore, the aim of this study was to determine the role of PSMA (i.e., ${ }^{18} \mathrm{~F}$ DCFPyL or ${ }^{68} \mathrm{Ga}-\mathrm{PSMA}-11$ ) PET/CT imaging in patients who experience BCP after RARP and to evaluate the sites of persistent disease on PSMA PET/CT. Methods: A total of 150 consecutive patients with BCP after RARP who underwent radiolabeled PSMA PET/CT imaging were retrospectively evaluated. BCP was defined as any detectable first serum PSA-value after RARP $(\geq 0.1 \mathrm{ng} / \mathrm{mL})$ at least 6 weeks after surgery, in the absence of an undetectable PSA-value after RARP. A multivariable logistic regression analysis was performed to identify predictors for the detection of metastases outside the prostatic fossa ( $\geq$ miN1) on PSMA PET/CT. Results: A PSMA PET/CT was performed at a median PSA-value of $0.60 \mathrm{ng} / \mathrm{mL}$ (interquartile range (IQR) 0.3-2.4) after a median period of 6 months (IQR 4-10) following RARP. In total, 101/150 patients (67\%) had lesions with PSMA-expression on PET/CT, of which 89/150 patients (59\%) had lesions with increased PSMA-expression sites outside the prostatic fossa. Moreover, 39/150 patients (26\%) had PSMA-positive lesions outside the pelvis. On multivariable analysis, higher PSA-values after $\operatorname{RARP}(p=0.004)$ and positive pathological lymph node status $(p=0.006)$ were independent predictors for $\geq \operatorname{miN1}$. Conclusion: In presence of BCP, a high proportion of patients already had metastatic disease to pelvic lymph nodes or showed evidence of distant metastases, as indicated by PSMA PET/CT. Higher PSA-levels after RARP and positive pathological lymph node status were significantly associated with metastases outside the prostatic fossa. In conclusion, in patients with BCP, PSMA PET/CT imaging is warranted to guide (salvage) treatment strategies. 
Keywords: biochemical persistence, prostate cancer, PSMA PET/CT imaging 


\section{INTRODUCTION}

In patients with localized prostate cancer ( $\mathrm{PCa})$, robot-assisted laparoscopic radical prostatectomy (RARP) is one of the main curative treatment options. After RARP, prostate-specific antigen (PSA)-measurements are performed to assess the oncological outcome. If PSA becomes undetectable (i.e., $<0.1 \mathrm{ng} / \mathrm{mL}$ ), it is assumed that patients are free of disease. Due to the half-life of PSA of 2 to 3 days (1), the serum PSA-level should become undetectable within approximately 6 weeks after RARP. However, a substantial percentage of patients (5-20\%) who undergo RARP, retain a persistently measurable PSA $(2,3)$. In literature, biochemical persistence $(B C P)$ of PSA after RARP has been associated with poor oncological outcome (4). Furthermore, BCP after RARP, defined in the EAU-guidelines as any detectable PSA-value $(\geq 0.1 \mathrm{ng} / \mathrm{mL})$ within $4-8$ weeks after surgery (5), is associated with higher tumor stages, higher Gleason scores, positive surgical margins and impaired overall survival $(3,4,6)$.

Several studies have shown that patients with BCP after RARP benefit less from local salvage therapies such as salvage radiation therapy to the prostatic fossa compared to patients with rising PSA-values after an initial undetectable PSA-value (i.e., biochemical recurrence (BCR)) $(7,8)$. This may indicate that these patients might already have been metastasized at the time of diagnosis or at the time of salvage therapy. Since the introduction of radiolabeled (either with ${ }^{68}$ Gallium and ${ }^{18}$ Fluoride) prostate-specific membrane antigen (PSMA) positron emission tomography/computed tomography (PET/CT), the detection of metastases has improved substantially compared to conventional imaging techniques, such as bone scintigraphy, CT-scan and magnetic resonance imaging, especially in patients with BCR of disease (9). With this modern imaging technique, a more confident localization of the PCa recurrences is possible, and adjustment of management decisions appears more frequently (10). For patients who do not benefit from local salvage therapies, among those with BCP after RARP, PSMA PET/CT might be 
the imaging tool of choice to guide management decisions and with this, optimize a personalized treatment approach.

Recently, Farolfi et al. (11) reported on a cohort of 191 patients with BCP after RARP, who underwent ${ }^{68} \mathrm{Ga}-\mathrm{PSMA} \mathrm{PET} / \mathrm{CT}$ with features of higher risk (median PSA-level of $1.1 \mathrm{ng} / \mathrm{mL}, 31 \%$ was pre-treated with androgen-deprivation therapy (ADT), $58 \%$ had an International Society of Urological Pathology (ISUP) Grade Group $\geq 4$ ). The aim of the present study however, was to determine the role of PSMA PET/CT imaging in hormone-naïve patients with early BCP after RARP. Particular attention was paid to the anatomical sites of PCa identified on PSMA PET/CT, stratified by the PSA-level at scan time. 


\section{MATERIALS AND METHODS}

This retrospective analysis was conducted by the Prostate Cancer Network the Netherlands, i.e., Amsterdam UMC, VU University Medical Center (VUmc) and the Netherlands Cancer Institute (NCl), in the period August 2016 and June 2020. Approval of the institutional review board of both hospitals was obtained for this study, meanwhile waiving the need to receive informed consent (VUmc2019.586 and IRBd20-016).

In the present study, we included 150 consecutive patients with BCP after RARP, who underwent either ${ }^{18} \mathrm{~F}-\mathrm{DCFPyL}$ or ${ }^{68} \mathrm{Ga}-\mathrm{PSMA}-11 \mathrm{PET} / \mathrm{CT}$. BCP was defined as any detectable first serum PSA-value after RARP $(\geq 0.1 \mathrm{ng} / \mathrm{mL})$ at least 6 weeks after surgery, in the absence of an undetectable PSA-value after RARP (5). Patients were excluded from analysis when using ADT or anti-hormonal therapy prior to PSMA PET/CT for BCP.

\section{Patient Data}

From all included patients, demographic data, biochemical data (PSA-level at BCP, PSA at the time of performing the PSMA PET/CT), radiological data (e.g., PSMA PET/CT results prior to RARP and at BCP) and pathological data (e.g., pathological tumor stage, prostatectomy specimen International Society of Urological Pathology (ISUP) Grade Group, surgical margin status, pathological lymph node status) were obtained and inserted in a comprehensive database. Time to first PSA-measurement was noted, as were the time between RARP and PSMA PET/CT and the PSA-values at the first sign of BCP. 


\section{PSMA PET/CT Imaging}

At VUmc, imaging was performed with a Philips Ingenuity TF (Philips Healthcare ${ }^{\circledR}$, the Netherlands/USA) PET/CT system, with ${ }^{18} \mathrm{~F}-\mathrm{DCFPyL}$ as tracer. ${ }^{18} \mathrm{~F}-\mathrm{DCFPyL}$ was synthesized via direct radiofluoration at the on-site cyclotron facility compliant to Good Manufacturing Practices (GMP) $(12,13)$. The median tracer dose given was $311 \mathrm{MBq}$ (interquartile range (IQR) 298-323 MBq). PET-images were acquired approximately 120 min after intravenous injection.

At $\mathrm{NCl}$, imaging was performed using a Philips Gemini TF-II or Vereos Digital PET/CT (Philips Healthcare ${ }^{\circledR}$, the Netherlands/USA), with either ${ }^{68} \mathrm{Ga}-\mathrm{PSMA}-11$ or ${ }^{18} \mathrm{~F}-\mathrm{DCFPyL}$, as tracers. ${ }^{68} \mathrm{Ga}-\mathrm{PSMA}-11$ was radiolabeled in-house using a fully automated system (Scintomics $\mathrm{GmbH}$, Germany). A fixed dose of 100 or (from September 2019) $150 \mathrm{MBq}$ was administered to patients as an intravenous bolus. Scanning commenced approximately 45 minutes after injection, with 3-4.5 minutes per bed position for pelvis/abdomen and 2-3 minutes per bed position for the

remainder of the scan range. ${ }^{18} \mathrm{~F}-\mathrm{DCFPyL}$ was administered as an intravenous bolus injection with a median dose of $201 \mathrm{MBq}($ IQR 192-208 MBq). Scanning commenced after an incubation period of approximately 60 minutes, with 2 minutes per bed position over the complete scan range.

All patients were asked to drink $1 \mathrm{~L}$ of water 60 min prior to tracer injection. No diuretics were administered. Immediately prior to the scans, patients were required to empty their urinary bladder.

PET-images were combined with either a low-dose CT-scan (120-140 kV, 40-80 mAs with dose modulation) or a diagnostic CT-scan (130kV, 110mAs) without contrast enhancement. All PET-images were corrected for scatter, decay, and random coincidences; attenuation correction was performed using CT images. 


\section{Image interpretation of PSMA PET/CT}

Scan interpretation was performed in participating centers by experienced nuclear medicine physicians. Scans were interpreted in clinical setting. A scan was considered 'positive' if at least one lesion was suggestive for PCa (i.e., focal, higher tracer uptake compared to the surrounding tissues, incompatible with physiological uptake, with anatomical substrate on CT). Following EAU-guidelines, and according to the PROMISE-criteria (14), loco-regional lymph node metastases were defined as lymph nodes in the true pelvis (miN1), whereas lymph nodes outside the surgical template were considered distant lymph node metastases (miM1a). Furthermore, it was assessed whether lesions with increased PSMA-expression were present in the bones (miM1b), and in visceral organs (miM1c) (5). Moreover, we assessed whether patients with metastatic disease (i.e., $\geq$ miN1) had unimetastatic or oligometastatic disease (2-5 metastases) $(15,16)$, and might therefore qualify for metastasis-directed therapy.

\section{Statistical analysis}

Numerical variables were summarized as medians and IQRs, while categorical variables were expressed as percentages (\%). Univariable and multivariable logistic regression analyses were performed with predefined variables, e.g., first PSA-value after RARP, pathological T-stage, ISUP Grade Group, surgical margin status and pathological lymph node status. Outcome variable was the presence of disease outside the prostatic fossa ( $\geq \mathrm{miN1}$ disease). Moreover, a multivariable logistic regression analysis to identify predictors for lesions with PSMA-expression outside the pelvis $(\geq \mathrm{miM} 1)$ was performed. Statistical analyses were performed using the Statistical Package for Social Sciences (SPSS, IBM; v25). Statistical significance was set at $p<0.05(17)$. 


\section{RESULTS}

\section{Patient characteristics}

In the present study, 150 patients with BCP after RARP were included. Patient characteristics are presented in Table 1. The median age of all patients at PSMA PET/CT was 68 years (IQR 62-72). The median PSA-value after RARP was $0.3 \mathrm{ng} / \mathrm{mL}$ (IQR 0.2-0.9) after a median follow-up of 99 days (IQR 72-125). A PSMA PET/CT was performed at a median PSA-value of $0.60 \mathrm{ng} / \mathrm{mL}$ (IQR 0.3-2.4) after a median period of 6 months (IQR 4-10) following RARP.

A pathological T-stage of $\geq \mathrm{pT} 3$ was found in $115 / 150$ patients $(77 \%)$, and $114 / 150$ patients (76\%) had ISUP Grade Group 3 or higher in the radical prostatectomy specimen. Moreover, 95/150 patients (63\%) had positive surgical margins. An extended pelvic lymph node dissection (ePLND) was performed in $117 / 150$ patients (78\%), of which $77 / 117$ patients $(66 \%)$ had evidence for malignant disease at histopathological evaluation ( $\mathrm{pN} 1)$. The median number of removed lymph nodes was 16 (IQR 11-22).

\section{PSMA PET/CT findings}

In 49/150 patients (33\%), no lesions with increased PSMA-expression were detected (i.e., negative PSMA PET/CT-scan) at a median PSA of $0.3 \mathrm{ng} / \mathrm{mL}$ (IQR 0.2-0.5). The remaining 101/150 patients (67\%) had a PSMA PET/CT with at least one lesion suggestive for PCa (i.e., 'positive') at a median PSA of $1.2 \mathrm{ng} / \mathrm{mL}$ (IQR 0.5-4.1; Mann-Whitney; $p<0.001)$. PSMA PET/CT positivity stratified for the PSA-value at the time of the scan is visualized in Figure 1. PSMA PET/CT positivity was observed in 24 of 57 (42\%) patients with PSA-values $<0.5 \mathrm{ng} / \mathrm{mL}$, in 25 of $37(68 \%)$ patients with PSA-values $0.5-<1.0 \mathrm{ng} / \mathrm{mL}$, in 12 of $14(86 \%)$ patients with PSA-values $1.0-<2.0 \mathrm{ng} / \mathrm{mL}$, and in 40 of $42(95 \%)$ patients with PSA-values $\geq 2.0 \mathrm{ng} / \mathrm{mL}$ (Table 2). 


\section{Anatomical sites of lesions with increased PSMA-expression on PSMA PET/CT}

From 101 patients with lesions with increased PSMA-expression on the PET/CT-scans, 62 patients $(61 \%)$ had detectable lesions limited to the pelvic area, 13 patients $(13 \%)$ had lesions

suggestive for PCa outside the pelvic area only, and 26 patients (26\%) had PSMA-expressing lesions both in/outside the pelvic area (Table 2). In total, 89 out of 101 patients (88\%) with tumor expressing PSMA on PET/CT-scan, had PSMA-avid sites outside the prostatic fossa (i.e., $\geq m i N 1$ ), whereas 39 out of 101 patients (39\%) had evidence of distant metastatic disease on PSMA PET/CT ( $\geq$ miM1; Figure 1). Of these 89 patients, 32 patients (36\%) had unifocal involvement, 31 patients (35\%) had oligometastatic disease and 26 patients $(29 \%)$ had polymetastatic disease.

\section{PSMA PET/CT imaging prior to RARP}

In 56/150 patients $(37 \%)$ a PSMA PET/CT was performed prior to surgery. The PSMA PET/CT was suggestive for pelvic lymph node metastases in $21 / 56$ patients (38\%), whereas $62 \%$ (35/46 patients) had a PSMA PET/CT-scan negative for metastatic disease. Of the 21 patients with PSMA-positive pelvic lymph nodes, 17 patients $(81 \%)$ had lesions suggestive for pelvic lymph node metastases on restaging PSMA PET/CT, of which 12 patients (57\%) had persistent pelvic lymph nodes and 5 patients $(24 \%)$ had recurrent pelvic lymph nodes. On the other hand, only 11/35 (31\%; Chi-square; $p<0.001)$ patients with a PSMA PET/CT negative for metastases prior to surgery had lesions suggestive for pelvic lymph node metastases on restaging PSMA PET/CT. No differences were found in the presence of distant metastases between patients with a negative pre-operative scan (9/35 patients; $26 \%$ ) and those with pelvic lymph node metastases on staging PSMA PET/CT (6/21 patients; 29\%; Chi-square; $p=0.82)$. 


\section{Logistic regression analysis}

On univariable logistic regression analysis, PSA-value at PSMA PET/CT $(p<0.001)$, first PSA-value after RARP ( $p=0.003)$, ISUP Grade Group 4-5 compared to $1-2$ in the radical prostatectomy specimen $(p=0.02)$ and pathological lymph node status $(p=0.001)$ were predictors of having recurrent disease outside the prostatic fossa (Table 3). In contrary, the time between RARP and PSMA PET/CT, pathological T-stage and surgical margin status were found not to have a predicting role. On multivariable logistic regression analysis, the first PSA-value after $\operatorname{RARP}(p=0.004)$ and pathological lymph node status $(p=0.006)$ were independent predictors for the detection of PCa deposits outside the prostatic fossa in patients with BCP after RARP (Table 3).

Secondly, we performed a multivariable logistic regression analysis on the detection of distant metastases (outside the pelvis) in patients with BCP after RARP. The first PSA-value after RARP $(p=0.002)$ was an independent predictor for distant metastases on PSMA PET/CT, whereas pathological lymph node status $(p=0.08)$ and surgical margin status were not $(p=0.37$; Table 3$)$. 


\section{DISCUSSION}

We retrospectively assessed the value of PSMA PET/CT imaging in 150 consecutive patients with BCP after RARP. Our results showed that $101 / 150$ included patients $(67 \%)$ had a 'positive' PSMA PET/CT-scan on BCP, of whom a remarkably high number of patients, 89/101 $(88 \%)$, showed evidence of disease outside the prostatic fossa ( $\geq$ miN1). Moreover, 39/101 patients (39\%) with a positive PSMA PET/CT-scan, had evidence of distant metastatic disease ( $\geq$ miM1). To our knowledge, limited data is available about the sites of localized disease with PSMA-expression on PET/CT, in patients with BCP after RARP.

When patients with BCP were stratified for PSA-value at the time of the PSMA PET/CT, $42 \%(24 / 57)$ and $68 \%(25 / 37)$ of patients with a PSA-value below $0.5 \mathrm{ng} / \mathrm{mL}$ and between 0.5 and $1.0 \mathrm{ng} / \mathrm{mL}$, respectively, had a positive PSMA PET/CT-scan, with lesions suggestive for PCa localizations (Table 2). These results are in line with those reported by Perera et al. in the systematic review on the oncological outcome of patients with BCR after radical prostatectomy, who underwent ${ }^{68} \mathrm{Ga}-\mathrm{PSMA}-$ based imaging for staging purposes (9). In that study, 33-48\% and $57 \%$ of scans performed at PSA-values below PSA $0.5 \mathrm{ng} / \mathrm{mL}$ and between 0.5 and $1.0 \mathrm{ng} / \mathrm{mL}$ after RARP, respectively, were positive for cancer recurrence (9). Using ${ }^{18} \mathrm{~F}-\mathrm{DCFPyL}$, Wondergem et al. reported similar percentages of positive PSMA PET/CT-scans at a PSA-value below 0.5 $\mathrm{ng} / \mathrm{mL}$ and between 0.5 and $1.0 \mathrm{ng} / \mathrm{mL}$ of $59 \%$ (17/29 patients) and $69 \%$ (20/29 patients), respectively (18).

The present study, however, reported on a specific subgroup of patients with BCP after RARP, i.e., those in whom the PSA-level did not become undetectable after RARP. In the literature, it was described that patients falling in this category might have a prognostically dismal outcome compared to patients who had an undetectable PSA-level postoperatively, before experiencing $\operatorname{BCR}(3,4,6)$. 
The majority of included patients with BCP in the present cohort had locally advanced PCa, positive lymph nodes and positive surgical margins. However, on multivariable logistic regression analysis, the first PSA-value after RARP $(p=0.004)$, and pathological lymph node status $(p=0.006)$ were statistically significant associated with disease outside the prostatic fossa ( $\geq$ miN1), whereas surgical margin status, ISUP Grade Group and pathological T-stage were not. Furthermore, assessing distant metastatic disease, the first PSA-value after RARP was found to be an independent predictor for the presence of distant metastases on PSMA PET/CT $(p=0.002)$. Apparently, the PSA-level at BCP remains an independent predictor of metastasized disease even if other powerful (pathological) predictors are assessed.

The results reported in the present study are partially comparable to a recent article evaluating ${ }^{68} \mathrm{Ga}-\mathrm{PSMA}-11 \mathrm{PET} / \mathrm{CT}$ in patients with $\mathrm{BCP}(11)$, with some essential differences. Farolfi et al. (11) reported on a cohort in which the median PSA-value at the time of the PSMA $\mathrm{PET} / \mathrm{CT}$ was $1.1 \mathrm{ng} / \mathrm{mL}$, remarkably higher compared to $0.6 \mathrm{ng} / \mathrm{mL}$ in the present study. Furthermore, considerably more patients had an ISUP Grade Group $\geq 4$ (58\%), compared to our study $(33 \%)$. Interestingly, in the study performed by Farolfi et al., almost one third of cases used ADT prior to surgery and/or PSMA PET/CT, while we included hormone-naïve patients, only. It is unknown to what extent hormonal treatment may have influenced PSMA-expression, but a flare phenomenon is possible (19). Lastly, Farolfi et al. reported on ${ }^{68} \mathrm{Ga}-\mathrm{PSMA}-11 \mathrm{PET} / \mathrm{CT}$ solely, while our cohort comprised also $37 \%{ }^{18} \mathrm{~F}$-DCFPyL PET/CT-scans. All these variables together may have resulted in a different proportion of metastases.

In studies assessing predictors of PSMA PET/CT-positivity in patients with BCR, PSA kinetics (e.g., PSA doubling time), were significantly associated with the detection rate of PSMA PET/CT (20). Unfortunately, due to nature and definition of BCP, dynamic metrics are of limited value in this cohort and were therefore not calculated. 
The present study is one of the few studies to report on patients with BCP after RARP in whom PSMA PET/CT was performed to locate the anatomical sites of lesions suggestive for PCa, stratified by PSA-level. A significant association was found between the level of PSA at the time of PSMA PET/CT and the extent of dissemination of disease. So, at higher PSA-levels, more voluminous and more distant metastatic disease were reported. This implicates that radiolabeled PSMA PET/CT imaging should be performed at the earliest signs of PSA-persistence, to guide potential salvage treatment (e.g., salvage radiation therapy, salvage lymph node dissection), metastasis-directed therapy in patients with oligometastatic disease, or, in patients with polymetastatic disease, early initiation of systemic treatment.

Interestingly, the surgical margin status was not a statistically significant predictor of metastatic disease neither on univariable nor on multivariable analysis. Furthermore, no association was found between the cumulative length of surgical margin positivity and the presence of (any) metastases on PSMA PET/CT. Therefore, also in patients with evident positive surgical margins who experience BCP, restaging PSMA PET/CT should be performed before initiation of salvage treatment.

In the present cohort, 21 patients had a PSMA PET/CT prior to surgery, that showed pelvic lymph node metastatic disease (miN1). On restaging PSMA PET/CT on BCP, 17/21 patients $(81 \%)$ had lesions suggestive for lymph node metastases in the pelvic area. When comparing the locations of these metastases on both the pre-operative and post-operative PSMA PET/CT, 12/21 patients $(57 \%)$ had persistent lymph nodes after surgery. This percentage is largely comparable to that reported by Farolfi et al. (11), who found persistent pelvic lymph nodes on PSMA PET/CT in $15 / 33$ patients $(45 \%)$.

Some limitations need to be addressed. Firstly, no histopathological verification of the PSMA PET/CT-detected lesions was obtained, due to comorbidity or ethical reasons. 
Consequently, false positive lesions on PSMA PET/CT might have influenced our results. However, the positive predictive value of PSMA PET/CT in (recurrent) PCa is reported being high (21). Therefore, we believe the number of false positive lesions on PSMA PET/CT in our cohort was low. Secondly, we do not report on the oncological follow-up of patients who underwent radiolabeled PSMA PET/CT imaging on BCP. In our series, patients underwent a wide variety of (salvage) treatments, such as salvage radiation therapy to the prostatic fossa, stereotactic ablative lymph node radiation therapy, salvage lymph node dissection and different hormonal treatments. Until now, no clear recommendations on treatment preference have been made using data from well performed randomized clinical trials. Moreover, different radiotracers and different scan protocols were used. This might have influenced the findings of the PSMA PET/CT-scans. Lastly, it might well be that the results derived from the multivariable analysis on the presence of metastases reported in this study may be skewed as a substantial proportion of our patients had 'no evidence of disease' on PSMA PET/CT. These patients with a 'negative for cancer' PSMA PET/CT may have a different distribution of (yet undetected) sites of PCa lesions than reported here, possibly resulting in a different outcome. 


\section{CONCLUSION}

The present study reports on the anatomical sites of PSMA PET/CT localized disease in patients who have biochemical persistence of PSA (BCP) after RARP. At BCP, a high proportion of patients already had metastatic disease to pelvic lymph nodes or distant sites, as indicated by PSMA PET/CT. Higher PSA-levels after RARP and positive pathological lymph node status were significantly associated with metastatic disease outside the prostatic fossa. In conclusion, in patients with BCP, PSMA PET/CT imaging is warranted to guide (salvage) treatment strategies. 


\section{DISCLOSURE}

Funding:

No funding was received for performing this study.

Conflict of interest:

The authors declare not to have any conflicts of interest.

\section{KEY POINTS}

Question:

What is the role of PSMA PET/CT imaging in patients who experienced BCP after RARP, and can we identify predictors for metastases on PSMA PET/CT?

\section{Pertinent findings:}

In this retrospective cohort study, we found that $59 \%$ of patients with BCP after RARP had metastases outside the prostatic fossa $(\geq \mathrm{miN} 1)$ on PSMA PET/CT. Higher PSA-levels after RARP and positive pathological lymph node status were significantly associated with metastatic disease outside the prostatic fossa.

Implications for patient care:

In patients with BCP, also in those with evident positive surgical margins, PSMA PET/CT imaging is warranted to guide (salvage) treatment strategies. 


\section{REFERENCES}

1. Lotan Y, Roehrborn CG. Clearance rates of total prostate specific antigen (PSA) after radical prostatectomy in African-Americans and Caucasians. Prostate Cancer Prostatic Dis. 2002;5:111-114.

2. Ploussard G, Staerman F, Pierrevelcin J, et al. Predictive factors of oncologic outcomes in patients who do not achieve undetectable prostate specific antigen after radical prostatectomy. J Urol. 2013;190:1750-1756.

3. Wiegel $T$, Bartkowiak $D$, Bottke $D$, et al. Prostate-specific antigen persistence after radical prostatectomy as a predictive factor of clinical relapse-free survival and overall survival: 10-year data of the ARO 96-02 trial. Int J Radiat Oncol Biol Phys. 2015;91:288-294.

4. Preisser F, Chun FKH, Pompe RS, et al. Persistent prostate-specific antigen after radical prostatectomy and its impact on oncologic outcomes. Eur Urol. 2019;76:106-114.

5. Mottet N, van den Bergh RCN, Briers E, et al. EAU - ESTRO - ESUR - SIOG Guidelines on Prostate Cancer 2020. European Association of Urology Guidelines. 2020 Edition. Vol presented at the EAU Annual Congress Amsterdam 2020. Arnhem, The Netherlands: European Association of Urology Guidelines Office; 2020.

6. Sood A, Keeley J, Palma-Zamora I, et al. Ten-year disease progression and mortality rates in men who experience biochemical recurrence versus persistence after radical prostatectomy and undergo salvage radiation therapy: A post-hoc analysis of RTOG 9601 trial data. Urol Oncol. 2020.

7. Bartkowiak D, Siegmann A, Bohmer D, Budach V, Wiegel T. The impact of prostate-specific antigen persistence after radical prostatectomy on the efficacy of salvage radiotherapy in patients with primary NO prostate cancer. BJU Int. 2019;124:785-791.

8. Fossati N, Karnes RJ, Colicchia M, et al. Impact of early salvage radiation therapy in patients with persistently elevated or rising prostate-specific antigen after radical prostatectomy. Eur Urol. 2018;73:436-444.

9. Perera M, Papa N, Roberts $\mathrm{M}$, et al. Gallium-68 prostate-specific membrane antigen positron emission tomography in advanced prostate cancer-updated diagnostic utility, sensitivity, specificity, and distribution of prostate-specific membrane antigen-avid lesions: a systematic review and meta-analysis. Eur Urol. 2020;77:403-417.

10. Calais J, Fendler WP, Eiber M, et al. Impact of (68)Ga-PSMA-11 PET/CT on the management of prostate cancer patients with biochemical recurrence. J Nucl Med. 2018;59:434-441. 
11. Farolfi A, Gafita A, Calais J, et al. (68)Ga-PSMA-11 positron emission tomography detects residual prostate cancer after prostatectomy in a multicenter retrospective study. J Urol. 2019;202:1174-1181.

12. Ravert HT, Holt DP, Chen Y, et al. An improved synthesis of the radiolabeled prostate-specific membrane antigen inhibitor, [(18) F]DCFPyL. J Labelled Comp Radiopharm. 2016;59:439-450.

13. Bouvet V, Wuest M, Jans HS, et al. Automated synthesis of [(18)F]DCFPyL via direct radiofluorination and validation in preclinical prostate cancer models. EJNMMI Res. 2016;6:40.

14. Eiber M, Herrmann K, Calais J, et al. Prostate cancer molecular imaging standardized evaluation (PROMISE): proposed miTNM classification for the interpretation of PSMA-ligand PET/CT. J Nucl Med. 2018;59:469-478.

15. Singh D, Yi WS, Brasacchio RA, et al. Is there a favorable subset of patients with prostate cancer who develop oligometastases? Int J Radiat Oncol Biol Phys. 2004;58:3-10.

16. Rao A, Vapiwala N, Schaeffer EM, Ryan CJ. Oligometastatic prostate cancer: a shrinking subset or an opportunity for cure? Am Soc Clin Oncol Educ Book. 2019;39:309-320.

17. Greenland S, Senn SJ, Rothman KJ, et al. Statistical tests, P values, confidence intervals, and power: a guide to misinterpretations. Eur J Epidemiol. 2016;31:337-350.

18. Wondergem M, Jansen BHE, van der Zant FM, et al. Early lesion detection with (18)F-DCFPyL $\mathrm{PET} / \mathrm{CT}$ in 248 patients with biochemically recurrent prostate cancer. Eur J Nucl Med Mol Imaging. 2019;46:1911-1918.

19. Aggarwal R, Wei X, Kim W, et al. Heterogeneous Flare in Prostate-specific Membrane Antigen Positron Emission Tomography Tracer Uptake with Initiation of Androgen Pathway Blockade in Metastatic Prostate Cancer. Eur Urol Oncol. 2018;1:78-82.

20. Pereira Mestre R, Treglia G, Ferrari M, et al. Correlation between PSA kinetics and PSMA-PET in prostate cancer restaging: A meta-analysis. Eur J Clin Invest. 2019;49:e13063.

21. Fendler WP, Calais J, Eiber M, et al. Assessment of 68Ga-PSMA-11 PET accuracy in localizing recurrent prostate cancer: a prospective single-arm clinical trial. JAMA Oncol. 2019;5:856-863. 
Table 1. Patient characteristics in presence of biochemical persistence (BCP) after robotassisted radical prostatectomy (RARP)

All included patients $(n=150)$

First PSA after RARP ( $\mathrm{ng} / \mathrm{mL})$; median (IQR)

$0.3(0.2-0.9)$

Time between RARP and first PSA-measurement (days); median (IQR)

$99(72-125)$

Age at time of the PSMA PET/CT (yrs); median (IQR)

$68(62-72)$

Time between RARP and PSMA PET/CT (months); median (IQR)

$6(4-10)$

$P S A$ at PSMA PET/CT ( $\mathrm{gg} / \mathrm{mL}) ;$ median (IQR)

$0.60(0.3-2.4)$

PSMA PET/CT tracer

${ }^{68}$ Gallium-PSMA-11

$94(63)$

${ }^{18}$ Fluoride-DCFPyL

$56(37)$

RARP T-stage; $\mathbf{n}(\%)$

pT2

$35(23)$

pT3a

$52(35)$

pT3b

$60(40)$

pT4

$3(2)$

RARP Grade Group according to ISUP; $\mathbf{n}(\%)$

5 (Gleason score $\geq 9$ )

$35(23)$

4 (Gleason score $=8$ )

$15(10)$

3 (Gleason score $4+3=7$ )

$64(43)$

2 (Gleason score $3+4=7$ )

$32(21)$

1 (Gleason score $3+3=6$ )

Surgical Margin status; $\mathbf{n}(\%)$

Negative

Positive

Missing

Cumulative length of surgical margin positivity (cm); median (IQR)

$1.0(0.3-2.0)$

RARP N-stage; $\mathbf{n}(\%)$

$\mathrm{pNx}$

pNO

$40(27)$

$\mathrm{pN} 1$

$77(51)$

Number of removed lymph nodes; median (IQR)

$16(11-22)$

PSA = prostate-specific antigen; RARP = robot-assisted laparoscopic radical prostatectomy; IQR = interquartile range; $\mathrm{PSMA}$ = prostate-specific membrane antigen; $\mathrm{PET}=$ positron emission tomography; $\mathrm{CT}=$ computed tomography; ISUP = International Society of Urological Pathology 
Table 2. Localization of lesions suggestive for prostate cancer on PSMA PET/CT imaging stratified per PSA-level

\begin{tabular}{|c|c|c|c|c|}
\hline & $\begin{array}{l}\text { PSA }<0.5 \\
(n=57)\end{array}$ & $\begin{array}{l}\text { PSA } 0.5-<1.0 \\
(n=37)\end{array}$ & $\begin{array}{l}\text { PSA } 1.0-<2.0 \\
(n=14)\end{array}$ & $\begin{array}{l}\text { PSA } \geq 2.0 \\
(n=42)\end{array}$ \\
\hline \multicolumn{5}{|l|}{ PSMA PET/CT findings; $\mathbf{n}$ (\%) } \\
\hline Negative for cancer & $33(57)$ & $12(32)$ & $2(14)$ & $2(5)$ \\
\hline Local recurrence of disease (miTr) & $4(7)$ & $5(14)$ & $0(0)$ & $3(7)$ \\
\hline Locoregional lymph node metastases (miN1) & $14(25)$ & $10(27)$ & $4(29)$ & $12(29)$ \\
\hline Distant lymph node metastases (miM1a) & $1(2)$ & $1(3)$ & $0(0)$ & $3(7)$ \\
\hline Bone- or visceral metastases (miM1b-M1c) & $1(2)$ & $2(5)$ & $0(0)$ & $0(0)$ \\
\hline Multiple locations & $4(7)$ & $7(19)$ & $8(57)$ & $22(52)$ \\
\hline \multicolumn{5}{|c|}{ PSMA PET/CT findings, stratified per location; $\mathbf{n}(\%)$} \\
\hline Negative & $33(58)$ & $12(32)$ & $2(14)$ & $2(5)$ \\
\hline Inside the pelvis (miTr/miN1) & $19(33)$ & $18(49)$ & $6(43)$ & $19(45)$ \\
\hline Outside the pelvis ( $\geq \mathrm{miM} 1)$ & $4(7)$ & $3(8)$ & $1(7)$ & $5(12)$ \\
\hline Inside and outside the pelvis & $1(2)$ & $4(11)$ & $5(36)$ & $16(38)$ \\
\hline \multicolumn{5}{|c|}{$\begin{array}{l}\text { PSMA PET/CT findings, disease outside prostatic } \\
\text { fossa ( } \geq \text { miN1); } \mathbf{n}(\%)\end{array}$} \\
\hline No & $37(65)$ & $17(46)$ & $2(14)$ & $5(12)$ \\
\hline Yes & $20(35)$ & $20(54)$ & $12(86)$ & $37(88)$ \\
\hline \multicolumn{5}{|c|}{$\begin{array}{l}\text { PSMA PET/CT findings, extent of metastatic disease; } \\
\text { n (\%) }\end{array}$} \\
\hline Negative/local recurrence (miTr) & $37(65)$ & $17(46)$ & $2(14)$ & $5(12)$ \\
\hline Unimetastastic disease & $15(26)$ & $10(27)$ & $2(14)$ & $5(12)$ \\
\hline Oligometastatic disease (2-5 metastases) & $5(9)$ & $8(22)$ & $6(43)$ & $12(28)$ \\
\hline Polymetastatic disease ( $>5$ metastases) & $0(0)$ & $2(5)$ & $4(29)$ & $20(48)$ \\
\hline
\end{tabular}


Table 3. Univariable and multivariable logistic regression analyses on the presence of metastases ( $\geq m i N 1$ and $\geq m i M 1)$ on PSMA PET/CT imaging

\begin{tabular}{|c|c|c|c|c|c|c|}
\hline & \multicolumn{2}{|c|}{$\begin{array}{l}\text { Univariable logistic } \\
\text { regression on } \geq \mathrm{miN} 1\end{array}$} & \multicolumn{2}{|c|}{$\begin{array}{l}\text { Multivariable logistic } \\
\text { regression on } \geq \mathrm{miN} 1\end{array}$} & \multicolumn{2}{|c|}{$\begin{array}{l}\text { Multivariable logistic } \\
\text { regression on } \geq \mathrm{miM} 1\end{array}$} \\
\hline & Odds ratio $(95 \% \mathrm{Cl})$ & $p$-value & Odds ratio $(95 \% \mathrm{Cl})$ & $p$-value & Odds ratio $(95 \% \mathrm{Cl})$ & $p$-value \\
\hline PSA at PSMA PET/CT & $2.12(1.40-3.22)$ & $<0.001$ & - & - & - & - \\
\hline First PSA-value after RARP & $2.17(1.30-3.62)$ & 0.003 & $2.26(1.29-3.94)$ & 0.004 & $1.43(1.14-1.80)$ & 0.002 \\
\hline Time between RARP and PSMA PET/CT & $1.01(0.98-1.04)$ & 0.69 & - & - & - & - \\
\hline \multicolumn{7}{|l|}{ RARP T-stage; n (\%) } \\
\hline pT2 & Reference & & Reference & & - & - \\
\hline рT3a & $1.19(0.50-2.81)$ & 0.69 & $0.49(0.12-1.93)$ & 0.31 & - & - \\
\hline pT3b & $1.89(0.81-4.43)$ & 0.14 & $0.89(0.21-3.75)$ & 0.88 & - & - \\
\hline \multicolumn{7}{|l|}{ RARP Grade Group according to ISUP } \\
\hline$\geq 4$ (Gleason score $\geq 8$ ) & Reference & & Reference & & - & - \\
\hline 3 (Gleason score $4+3=7$ ) & $0.78(0.36-1.71)$ & 0.54 & $1.38(0.48-3.97)$ & 0.55 & - & - \\
\hline $1-2($ Gleason score $3+3=6$ and $3+4=7)$ & $0.34(0.14-0.82)$ & 0.02 & $0.38(0.10-1.45)$ & 0.16 & - & - \\
\hline \multicolumn{7}{|l|}{ Surgical Margin Status } \\
\hline Negative & Reference & & Reference & & Reference & \\
\hline Positive & $0.52(0.26-1.07)$ & 0.07 & $0.39(0.13-1.14)$ & 0.09 & $0.64(0.25-1.68)$ & 0.37 \\
\hline $\begin{array}{l}\text { Cumulative length of surgical margin } \\
\text { positivity }\end{array}$ & $1.16(0.89-1.53)$ & 0.28 & - & - & - & - \\
\hline \multicolumn{7}{|l|}{ Pathological lymph node status } \\
\hline pNO & Reference & & Reference & & Reference & \\
\hline $\mathrm{pN} 1$ & $3.75(1.68-8.37)$ & 0.001 & $4.10(1.49-11.3)$ & 0.006 & $2.64(0.91-7.69)$ & 0.08 \\
\hline
\end{tabular}




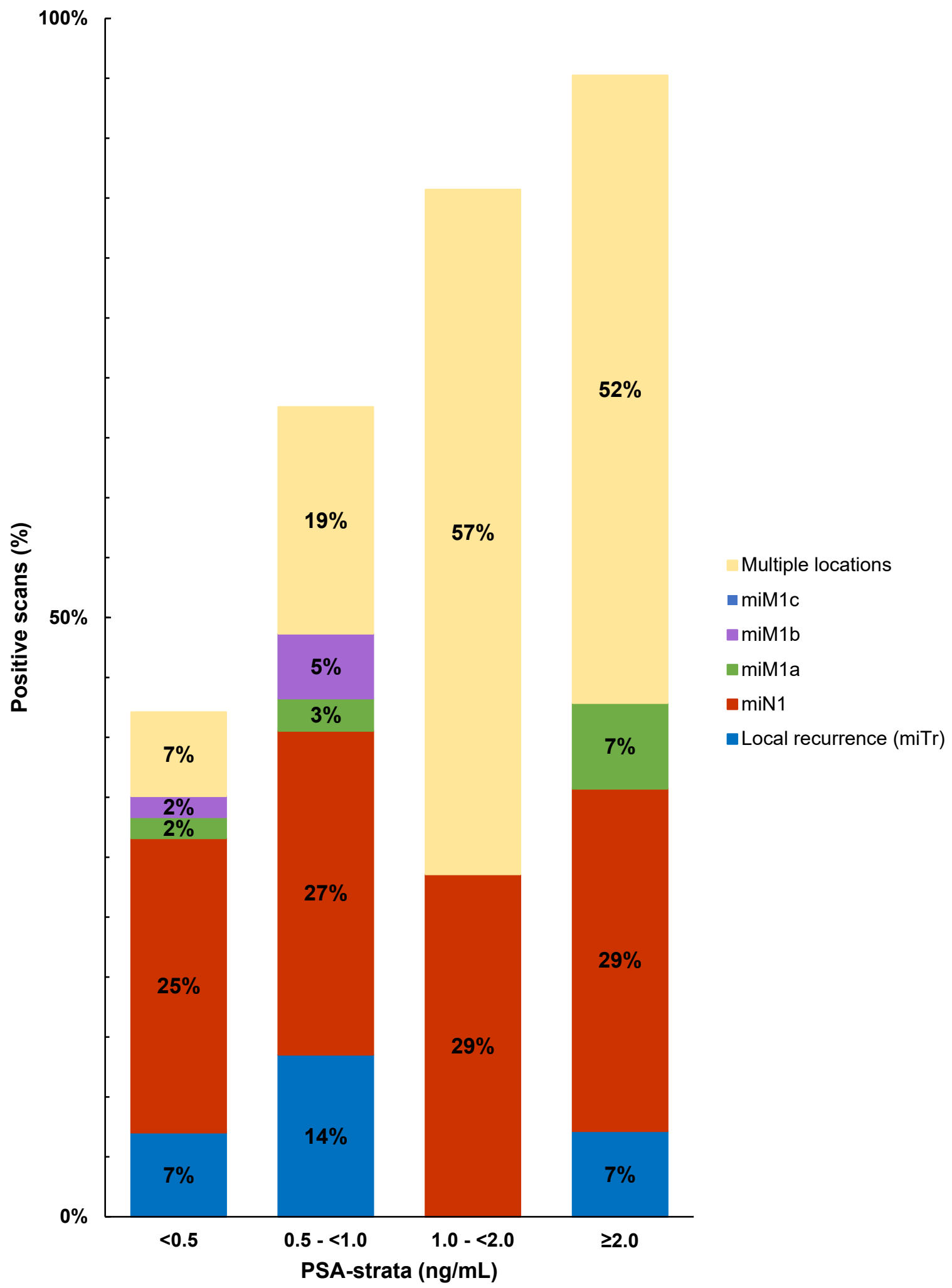

Figure 1. Sites of disease using PSMA PET/CT imaging, stratified per PSA-level at the time of PSMA PET/CT in patients with biochemical persistence after RARP 
Graphical Abstract

Biochemical Persistence of PSA after RARP: Tumor localizations using PSMA PET/CT

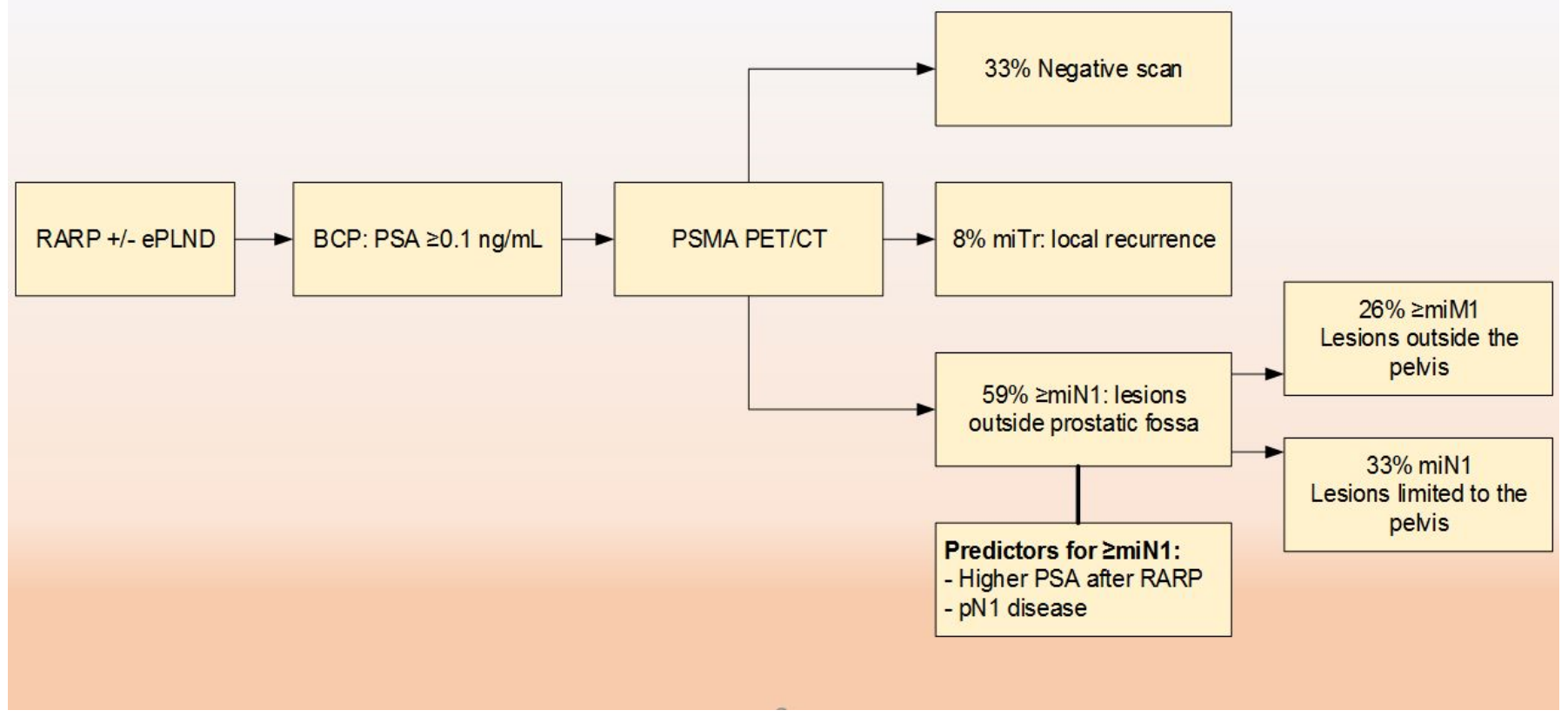

